\title{
Co-targeting the IGF system and HIF-1 inhibits migration and invasion by (triple-negative) breast cancer cells
}

\author{
M Mancini ${ }^{1}$, M B Gariboldi ${ }^{1}$, E Taiana ${ }^{1}$, M C Bonzi ${ }^{1}$, I Craparotta ${ }^{1}$, M Pagin ${ }^{1}$ and E Monti ${ }^{\star}, 1$ \\ ${ }^{1}$ Division of Biomedical Research, Department of Theoretical and Applied Sciences, University of Insubria, via A. da Giussano 10, \\ Busto Arsizio, Varese 21052, Italy
}

Background: Metastatic triple-negative breast cancer is mostly incurable, due to lack of suitable drug targets. The insulin-like growth factor (IGF) system could provide such a target, and IGF-1 receptor (IGF-1R)-directed agents are already available, but seem unable to control all the complexities of the system, including crosstalk with hypoxia-inducible pathways.

Methods: Migration of triple-negative MDA-231 breast cancer cells and its modulation by IGFs, the IGF-1R inhibitor NVP-AEW541 and the IGF-2-sequestering monoclonal antibody MAB292 were assessed by the scratch wound healing and Boyden chamber assays; the effect of topotecan (inhibiting hypoxia-inducible factor-1 (HIF-1)) under hypoxia was also evaluated. Constitutive as well as drug-modulated levels of components of the IGF and HIF-1 pathways were evaluated by western blotting and gPCR.

Results: IGF-induced migration of MDA-231 cells was not abrogated by the IGF-1R inhibitor NVP-AEW541, whereas IGF-2 sequestration by MAB292 significantly reduced cell migration. Under hypoxia, topotecan was also effective, likely by reducing HIF-1-induced IGF-2 release. Simultaneous targeting of IGF-1R and IGF-2 or HIF-1 completely abolished cell migration.

Conclusions: IR activation may account for the failure of NVP-AEW541 to suppress MDA-231 cell migration. Ligand-targeting compounds, or co-inhibition of the IGF and HIF-1 systems, may prevent activation of compensatory signalling, thereby providing a valuable addition to IGF-1R inhibitor-based therapies.

Despite major advances in early detection and treatment, breast cancer remains the most frequently diagnosed cancer and the leading cause of cancer death in women worldwide. Strategies targeting the primary tumour have markedly improved in the past decade, but systemic treatments to prevent metastasis are less effective, and about $20 \%-30 \%$ of patients develop metastatic disease (metastatic breast cancer (MBC)). Several targeted agents have been introduced into chemotherapeutic regimens for MBC in recent years (Beslija et al, 2009; Sánchez-Muñoz et al, 2009), and many others are in preclinical or early clinical stages of development, mostly in combination with cytotoxic chemotherapy (Alvarez et al, 2010). However, once the disease metastasises to distant areas of the body, typically to the lung, bone, liver and central nervous system, it remains incurable, with a median survival between 2 and 4 years, depending on the subtype (Guarneri and Conte, 2009; Alvarez et al, 2010).
Although representing only about $15 \%$ of all diagnosed breast cancer, the triple-negative subtype (TNBC), defined as negative for oestrogen receptor (ER), progesterone receptor (PR) and human epidermal growth factor receptor 2 (HER2) expression, accounts for a disproportionate share of deaths, due to aggressive tumour behaviour, poor prognosis and lack of targeted therapies. TNBC generally occurs in younger women and is associated with a high risk of distant recurrence (Reis-Filho and Tutt, 2008). Thus, the as yet unmet need of effective agents for the management of metastatic TNBC (mTNBC) is fuelling the search for novel therapeutic targets (Liedtke and Kiesel, 2011).

A large body of evidence indicates that the insulin/insulin-like growth factor (IGF) pathway is strongly implicated in breast cancer (Sachdev and Yee, 2001). The system consists of three ligands (IGF-1, IGF-2 and insulin); their cell surface receptors IGF-1 receptor (IGF-1R), IGF-2 receptor (IGF-2R, also known as the 
mannose 6-phosphate receptor M6P/IGF-2R) and two isoforms of the insulin receptor IR-A and IR-B, as well as hybrid IR/IGF-1Rs; six high-affinity binding proteins (IGFBP-1 to IGFBP-6) and their proteases (Sachdev and Yee, 2007; Pollak, 2008b). High IGF-1 plasma levels are a risk factor for breast cancer in premenopausal women (Hankinson et al, 1998), and significantly higher serum IGF-1 levels have been found in breast cancer patients as compared with healthy controls (Helle, 2004; Martin and Baxter, 2011). Loss of heterozigosity at the IGF2 gene has long been observed as a frequent occurrence in human breast cancer samples (McCann et al, 1996) and IGF-2 binding to IGF-1R and IR-A was found to facilitate activation of cell survival pathways (Richardson et al, 2011). The role of the IGF system in breast cancer has best been described in ER-positive tumours and cell lines, where it cooperates with the ER in promoting tumour growth and progression, while hampering the success of endocrine therapy (Zhang et al, 2011). Interestingly, IGF-1 has also been recently reported to promote proliferation and survival of TNBC cells (Davison et al, 2011); in addition, a role has recently been described for IGF signalling in tumour metastasis and invasion (Sachdev et al, 2010; Zhang et al, 2010; Saldana et al, 2013), increasing the appeal of the IGF system as a potential target for novel therapies directed at the ill-fated mTNBC subtype. Monoclonal antibodies and kinase inhibitors targeting IGF-1R have been the object of several clinical trials in different tumour types, but somehow the preclinical promise has failed to translate into therapeutic efficacy so far. This is possibly due to the redundancy and plasticity of the IGF system, whereby IR activation can compensate for IGF-1R disruption when this is specifically targeted (Zhang et al, 2007), especially when IGF-2 is the main stimulus acting on cancer cells. Most human cancers overexpress both IGF$1 \mathrm{R}$ and IR, leading to the formation of hybrid IGF-1R/IRs, and IGF-2 is a known ligand for all three receptors, displaying a far greater affinity for IR holoreceptors and IGF-1R/IR hybrids than IGF-1 (Pandini et al, 1999; Belfiore, 2007). The role played by IGF2 might be particularly relevant to therapeutic failure, as release of this factor from tumour cells can be driven by hypoxia-inducible factor-1 (HIF-1) as part of the adaptive response to reduced oxygen levels. Approximately $25-40 \%$ of all invasive breast cancer samples are hypoxic and express high levels of HIF- $1 \alpha$, the $\mathrm{O}_{2}$-labile HIF-1 $\alpha$-subunit required for transcriptional activation (Vaupel et al, 1991; Vleugel et al, 2005). Thus, the well-known ability of HIF-1 to promote aggressive breast cancer disease might depend, at least in part, on the recruitment of IGF-2-dependent signalling; in addition, IGF-2 release by HIF-1 might account for the limited clinical success of IGF-1R-targeted agents.

The present study was aimed at investigating the role played by IGF-2 in MDA-231 TNBC cell migration by emphasising its interactions with non-IGF-1R receptors. To this aim, we first verified that IGF-2-induced MDA-231 cell migration is associated with both IGF-1R and IR activation and that blocking IGF-1R with the selective catalytic inhibitor NVP-AEW541 (García-Echeverría et al, 2004) was unable to suppress either cell migration or IR phosphorylation, whereas sequestering IGF-2 with the neutralising antibody MAB292 dramatically reduced both, and completely abrogated them in the presence of NVP-AEW541. In addition, we also observed that HIF- $1 \alpha$ stabilisation reproduced, albeit on a lesser scale, the modifications observed in the presence of exogenous IGF-2 (i.e., increased cell migration and IGF-1R/IR phosphorylation), which could be prevented by adding the HIF-1 inhibitor topotecan and totally abolished by the topotecan/NVPAEW541 combination.

Overall, our data support the hypothesis that IR activation by IGF-2 may account for the failure of IGF-1R only-targeting agents to suppress TNBC cell migration in vitro, suggesting that it may also be involved in the disappointing clinical activity exhibited by such agents. Ligand-targeting compounds, or concomitant inhibition of the IGF system and HIF- $1 \alpha$, may prevent activation of compensatory signalling through IR or hybrid IR/IGF-1R and may provide an alternative or complementary strategy for the management of metastatic breast cancer in general and of TNBC in particular.

\section{MATERIALS AND METHODS}

Cell lines, reagents and drugs. Three human breast cancer cell lines were analysed: T47D and MCF-7 $\left(\mathrm{ER}^{+}, \mathrm{PR}^{+}, \mathrm{HER} 2^{-}\right)$and MDA-231 (ER $\left.\alpha^{-}, \mathrm{PR}^{-}, \mathrm{HER} 2^{-}\right)$, obtained from the American Type Culture Collection (ATCC, Rockville, MD, USA) through the Tumour Microenvironment Laboratory (TML, SAIC-NCI Frederick, MD, USA), and authenticated by morphological inspection, growth curve analysis and short tandem repeat profiling, using the Promega PowerPlex 1.2 system (Promega, Madison, WI, USA) and the Applied Biosystems Genotyper 2.0 software (Applied Biosystems, Foster City, CA, USA) for analysis of the amplicons. An additional triple-negative cell line, MDA-468, was obtained from the same source and used to replicate some of the experiments for confirmation purposes. Cells were routinely cultured as monolayer at $37^{\circ} \mathrm{C}$ and $5 \% \mathrm{CO}_{2}$ in RPMI (or DMEM in the case of MDA468) medium supplemented with $5 \%$ fetal bovine serum (FBS, Euroclone, Milan, Italy) and $1 \%$ antibiotics (penicillin/streptomycin, Sigma Aldrich, Milan, Italy).

NVP-AEW541 was kindly provided by Novartis Pharma (Basel, Switzerland): a DMSO stock solution $(10 \mathrm{mM})$ was prepared and stored at $-20{ }^{\circ} \mathrm{C}$. MAB292, rhIGF-1 and rhIGF-2 were purchased from R\&D Systems (Minneapolis, MN, USA): stock solutions were prepared following the manufacturer's instructions and stored at $-20^{\circ} \mathrm{C}$. Topotecan (NSC 609699) was kindly provided by the TML (SAIC-NCI Frederick, MD, USA). Working dilutions of all drugs were prepared in culture media immediately before use.

Quantitative RT-PCR. To analyse the components of the IGF and HIF systems at the transcript level, cells were seeded in dishes, allowed to grow for $24 \mathrm{~h}$ under normoxia $\left(\mathrm{pO}_{2} 21 \%\right)$ and subsequently incubated under hypoxia $\left(\mathrm{pO}_{2} 1 \%\right)$ for an additional $24 \mathrm{~h}$. Transcription of HIF-1 target genes (IGF2, VEGFA, PDK1) was also assessed following treatment with a subtoxic concentration of topotecan $(250 \mathrm{~nm})$ during the $24 \mathrm{~h}$ of hypoxia. Total RNA was extracted following the manufacturer's instructions (RNeasy kit, Qiagen, Venlo, Netherlands) and quantitated (ND-1000, NanoDrop, Thermo Fisher Scientific, Waltham MA, USA); $250 \mathrm{ng}$ (in $10 \mu \mathrm{l}$ ) of mRNA were added to $15 \mu \mathrm{l}$ of RT solution (High Capacity cDNA Reverse Transcription Kit, Applied Biosystems) and retro-transcribed following the optimised protocol. The cDNA thus obtained was used in a real time-PCR reaction: $2.5 \mathrm{ng}(3.75 \mu \mathrm{l})$ of cDNA were added with $21.25 \mu \mathrm{l}$ of a solution containing $2 \times$ Master Mix (TaqMan or SYBR, Applied Biosystems), $3 \mu \mathrm{M}$ reverse and forward primers (final concentration $300 \mathrm{~nm}$, custom made, Integrated DNA Technologies, Coralville, IA, USA), $20 \times$ eukaryotic $18 \mathrm{~S}$ rRNA endogenous control (VIC/TAMRA probe, Applied Biosystems) and $\mathrm{H}_{2} \mathrm{O}$. The run was performed following the protocol provided by the manufacturer, and the results were analysed by the ABI7300 software (Applied Biosystems). Primer sequences are reported in Supplementary Table S1.

Immunoblot analysis. To analyse the constitutive levels of the different components of the IGF and HIF systems, as well as the effects of growth factors and drugs on the IR/IGF-1R signalling pathway, cells were grown for $24 \mathrm{~h}$ and then treated with NVPAEW541 $(1 \mu \mathrm{M})$ and/or MAB292 $\left(5 \mu \mathrm{g} \mathrm{ml}^{-1}\right)$ for $24 \mathrm{~h}$. Cells were exposed to rhIGF-1 or rhIGF-2 $\left(50 \mathrm{ng} \mathrm{ml}^{-1}\right)$ during the last $15 \mathrm{~min}$ before harvesting, following 6-h starvation in serum-free medium. The effects of hypoxia (1\%) on the insulin/IGF system 
were assessed on cells grown for $24 \mathrm{~h}$ at $21 \% \mathrm{pO}_{2}$ and shifted to hypoxia for an additional $24 \mathrm{~h}$. NVP-AEW541 $(1 \mu \mathrm{M})$, MAB292 $\left(5 \mu \mathrm{g} \mathrm{ml}^{-1}\right)$ and Topotecan $(250 \mathrm{~nm})$ were added, singly or in combination, during the $24 \mathrm{~h}$ of hypoxia. Cell lysates were prepared in RIPA buffer containing protease inhibitor cocktail (Sigma Aldrich); protein phosphatases were inhibited by adding phenylmethylsulfonylfluoride (1 mM) and sodium orthovanadate $\left(\mathrm{Na}_{3} \mathrm{VO}_{4}, 1 \mathrm{~mm}\right)$ to the lysis buffer. Protein concentration was determined using assay kits based on the Bradford method (BioRad Life Science, Hercules, CA, USA), and equivalent amounts of total cell lysates $(100 \mu \mathrm{g})$ were added with sample buffer $2 \times$ (Laemmli, Sigma Aldrich), separated by $7 \%$ or $10 \%$ acrylamide SDS/PAGE under denaturing conditions and transferred onto PVDF membranes. Membranes were incubated overnight with the following primary antibodies: anti-phospho-IGF-1R $\beta$ (Tyr1316), anti-IGF-1R $\beta$, anti-phospho-IR $\beta$ (Tyr1361), anti-IR $\beta$, anti-phospho-Akt (Ser473) and anti-Akt (all from Cell Signalling Technology, Danvers, MA, USA); anti-IGF-2R (Abcam, Cambridge, UK); and anti HIF- $1 \alpha$ (Novus Biologicals, Littleton, CO, USA). An antiactin antibody (Sigma Aldrich) was used as a control. Membranes were then incubated with secondary anti-rabbit or anti-mouse antibody conjugated to horseradish peroxidase (Amersham, GE Healthcare Bio-Sciences, Pittsburgh, PA, USA). Immunoreactive bands were revealed by Enhanced Chemiluminescence Western Blotting Detection reagents (Amersham and Pierce) and visualised on Hyperfilm ECL (Amersham).

ELISA assay. The release of IGF-2 in culture media was evaluated under normoxic and hypoxic conditions. Cells were seeded onto six-well plates and allowed to grow for $24 \mathrm{~h}$ before starving and incubation at different oxygen levels $\left(\mathrm{pO}_{2} 21 \%\right.$ or $\left.1 \%\right)$. Supernatants were collected $24 \mathrm{~h}$ later and stored at $-80{ }^{\circ} \mathrm{C}$ or immediately quantitated using a specific ELISA kit (Insight Genomics, Falls Church, VA, USA), according to the manufacturer's protocols.

Flow cytometry. Membrane expression of IGF-1R, IGF-2R and IR was evaluated in all cell lines. Cells were seeded in six-well plates and allowed to grow for $48 \mathrm{~h}$; they were subsequently collected, counted and incubated for $1 \mathrm{~h}$ at $4{ }^{\circ} \mathrm{C}$ with specific conjugated antibodies (IGF-1R/PE, IGF-2R/FSC and IR/PE, R\&D Systems) as well as IgG isotype control antibodies (R\&D Systems). Red (PE) and green (FSC) fluorescence was then read using a Guava easyCyte (EMD Millipore, Billerica, MA, USA) flow cytometer. Background fluorescence, assessed in IgG isotype controls, was subtracted to the corresponding samples during analysis, and the percentage of fluorescent cells was calculated.
Scratch wound-healing assay. To evaluate the effect of the different compounds on migration of the three cell lines, cells were seeded at high density onto specific supports ( $\mu$-dish Culture Inserts, Idibi GmbH, Planegg/Martinsried, Germany) and allowed to grow for $24 \mathrm{~h}$. The inserts were then removed, the medium was replaced with serum-free medium, with or without the different compounds, and the dishes were incubated in different conditions of oxygenation $\left(\mathrm{pO}_{2} 21 \%\right.$ or $\left.1 \%\right)$. Pictures of the wound scratch were taken at the time of removal (T0) and at regular time intervals for a total of $48 \mathrm{~h}$. The experiments were repeated twice.

Boyden chamber assay. To further assess the migration of MDA231 cells, we also performed the Boyden chamber assay. Boyden chambers (Cell Biolabs Inc., San Diego, CA, USA) were preincubated for $30 \mathrm{~min}$ at $37^{\circ} \mathrm{C}$ in 24 -well plates containing $600 \mu \mathrm{l}$ of culture medium and FBS $(1: 1)$. Cells, previously starved for $24 \mathrm{~h}$, were added on top of the chambers, treated with growth factors and/or drugs at different oxygen levels $\left(\mathrm{pO}_{2} 21 \%\right.$ or $\left.1 \%\right)$ and allowed to grow and migrate for 24 or $48 \mathrm{~h}$. At the specified end points, the chambers were removed from the plates, fixed and stained in two steps (Eosin + AzurA/AzurB, 2 min each) and photographed. Analysis did not involve counting the number of migrating cells but only assessing visible differences between the treatments. The experiments were repeated twice.

\section{RESULTS}

In vitro assessment of IGF-stimulated migration and analysis of IGF system components in three human breast cancer cell lines. IGF-stimulated migration of MCF-7, T47D and MDA-231 cells was assessed using the scratch wound-healing and Boyden chamber assays. In the absence of stimulation, MCF-7 and T47D were unable to migrate, and even the addition of exogenous rhIGF1 or -2 did not appreciably modify their behaviour; in contrast, MDA-231 cells, derived from a metastatic carcinoma, intrinsically exhibited some degree of migration, which was substantially increased following stimulation with rhIGFs (Figure 1).

We then assessed the baseline levels of some major components of the IGF system, in order to evaluate their potential roles in the different migratory behaviour exhibited by the three cell lines. The results of PCR experiments (Supplementary Figure S1) indicate that IGF-2, IGF-1R and IGF-2R mRNAs are present in all cell lines, albeit at different levels: surprisingly, highly invasive MDA-231 showed lower levels of IGF-1R and higher levels of IGF-2R, as compared with both MCF-7 and T47D. IGF-1 mRNAs were undetectable in all cell lines and are not shown. Specific analysis of the two IR variants confirmed the results reported in the literature (Pollak, 2012): all the
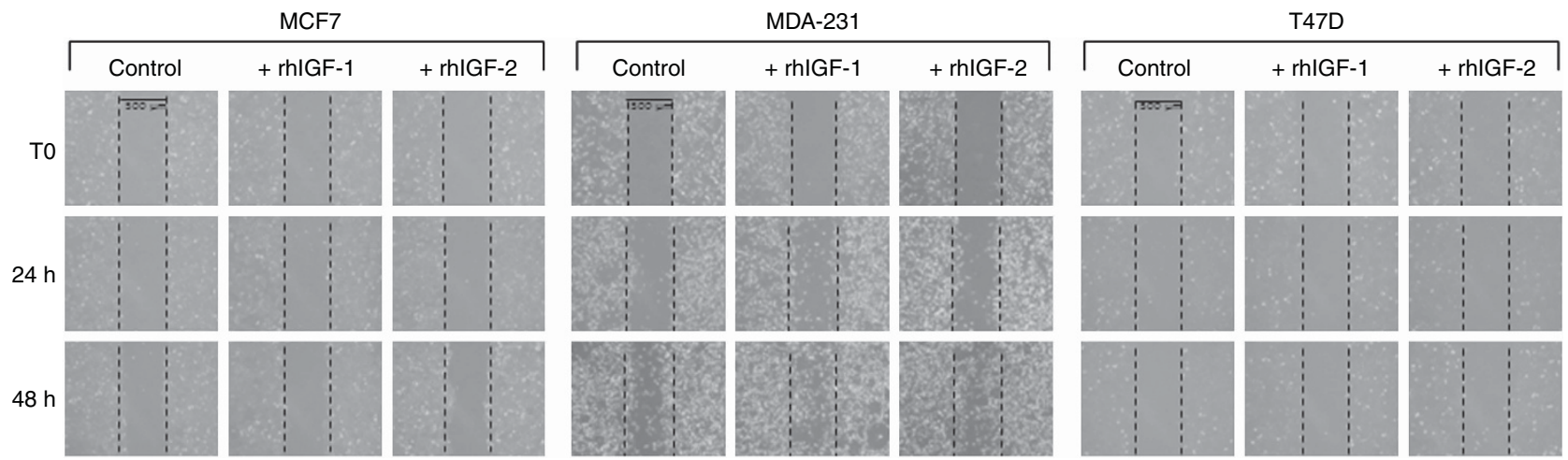

Figure 1. Migratory behaviour of MCF-7, MDA-231 and T47D cells under normoxic conditions $\left(\mathrm{pO}_{2} 21 \%\right.$ ), as assessed by the scratch woundhealing assay. Cells were grown in specific supports for $24 \mathrm{~h}$, then the inserts were removed and the medium was replaced with serum-free medium, with or without rhIGF-1 or rhIGF2 $\left(50 \mathrm{ng} \mathrm{ml}^{-1}\right)$. Pictures were taken at the time of insert removal (T0) and at regular time intervals for a total of $48 \mathrm{~h}$. 
cell lines tested expressed both the A and B variants of the receptor, with a predominance of the former; more specifically, MDA-231 cells were found to possess the highest levels of IR-A mRNA.

However, when considering protein levels, differences among cell lines were somewhat less evident: secreted IGF-2 in the extracellular medium was comparable in the three cell lines (Supplementary Figure S2, panel a), in spite of remarkable differences in transcript; IGF-1 expression was not investigated further, in view of the observed absence of transcript. Membrane expression of IGF-1R, as determined by flow cytometry, was consistent with transcript levels, whereas expression of IGF-2R and IR did not vary among cell lines (Supplementary Figure S2, panel b); however, it should be emphasised that IR-A and IR-B levels could not be determined separately, due to the lack of antibodies able to discriminate between the two isoforms.

IGF-1R and IR activation following exposure to exogenous IGFs was assessed by western blotting, based on the levels of receptor phosphorylation, and is shown in Figure 2 (densitometric analyses are reported in Supplementary Figures S3, S4 and S5). In unstimulated cells, both receptors exhibited a minimal degree of phosphorylation, which was markedly enhanced in response to the addition of rhIGFs to the culture medium. More specifically, as expected, IGF-1R was activated by both ligands in all three cell lines tested; in MDA-231, but not in the other cell lines, IR was also relevantly activated by rhIGF-2 and, to a lesser extent and rather more surprisingly, rhIGF-1. Exposure to the selective IGF-1R inhibitor NVP-AEW541 predictably abrogated IGF-1R phosphorylation in all cell lines but did not affect IR activation.

The selective IGF-1R inhibitor NVP-AEW541 is only partially effective in inhibiting cell migration following exposure to rhIGFs. As shown in Figure 3A and B, exposure to NVP-AEW541 partially abrogated the migration potential of MDA-231 cells, as assessed by both the scratch wound healing and the Boyden chamber migration. As expected, the inhibitor appeared to be more effective in reducing rhIGF-1 than rhIGF-2-induced migration. The fact that migration was not completely abolished could be explained by the inability of NVP-AEW541 to prevent IR activation (shown in Figure 2) and to block the related downstream pathway, as indicated by the presence of some residual Akt phosphorylation following exposure to rhIGF-2 in the presence of NVP-AEW541 in MDA-231 cells, in spite of a near-complete block of IGF-1R phosphorylation (Figure 4A; densitometric analyses are reported in Supplementary Figure S6). Interestingly, NVPAEW541 ehxibited some (slight) anti-migratory effect even on unstimulated cells, which might be explained by the presence of a low, baseline level of constitutive IGF-1R activation, as also suggested by the western blotting results shown in Figure 4A.

Enhancing the effects of NVP-AEW541 on cell migration by inhibiting IGF-2 signalling with the specific monoclonal antibody MAB292. To further demonstrate that IR activation by
rhIGF-2 accounts, at least in part, for the lack of efficacy of NVP-AWE541 in inhibiting the migration of MDA-231 cells, receptor-ligand interaction was antagonised using a neutralising antibody (MAB292) that binds and sequesters IGF-2. As shown in Figure 4A, MAB292 caused a decrease in the phosphorylation of both IGF-1R and IR in MDA-231 cells following stimulation with rhIGF-2; furthermore, rhIGF-2-dependent IR phosphorylation, as well as downstream Akt phosphorylation, was totally abolished when NVP-AEW541 and MAB292 were added simultaneously to the culture medium. This last observation can probably be explained by the inability of the IGF-sequestering antibody to counteract the baseline constitutive activity of IGF-1R observed above.

In agreement with the role played by IR in MDA-231 cell migration in response to rhIGF-2, results obtained in the scratch wound-healing (Figure 4B) and Boyden chamber assays (Figure 4C) indicate that, while IGF-1R inhibition alone was insufficient in preventing IGF-2-dependent migration, blocking the interaction of the growth factor with both signalling receptors using MAB292 showed some effect, which resulted in an almost complete abrogation of the migratory capacity of MDA-231 cells when NVP-AEW541 and MAB292 are added simultaneously. To confirm these findings on an additional TNBC cell line, the scratch wound-healing assay was also performed on MDA-468 cells stimulated with rhIGF-2, in the presence or absence of MAB292, NVP-AEW541 or both; the results are shown in Supplementary Figure S7 and were similar to those obtained in MDA-231 cells.

HIF- $1 \alpha$ stabilisation decreases the anti-migratory effect of NVP-AEW541. Hypoxia has been shown to cause an increase in endogenous IGF-2 release, owing to transcriptional activation of the corresponding gene by HIF-1 (Feldser et al, 1999; Semenza, 2012). Thus, our study was extended to examine the mechanism of IGF-2-dependent migration, and its response to NVP-AEW541, in hypoxic MDA-231 cells.

In agreement with literature data, secreted IGF-2 in the extracellular medium was significantly increased from $50.5 \pm$ 11.53 to $86.0 \pm 0.95 \mathrm{pg} \mathrm{ml}^{-1}(P<0.05)$ following hypoxic incubation. Western blotting analysis indicates that both IGF-1R and IR were partially phosphorylated under hypoxia, even in the absence of exogenous growth factor stimulation, and that NVP-AEW541 decreased IGF-1R phosphorylation, while leaving IR phosphorylation virtually unaffected; in contrast, treatment with MAB292 reduced the phosphorylation of both receptors (Figure 5A; densitometric analyses are reported in Supplementary Figure S8). These observations correlate with the migratory behaviour of hypoxic MDA-231 cells, in that migration was almost completely blocked by MAB292, whereas NVP-AEW541 only caused partial inhibition (Figure $5 \mathrm{~B}$ and $\mathrm{C}$ ), similarly to what had been observed following exposure to exogenous rhIGF-2.
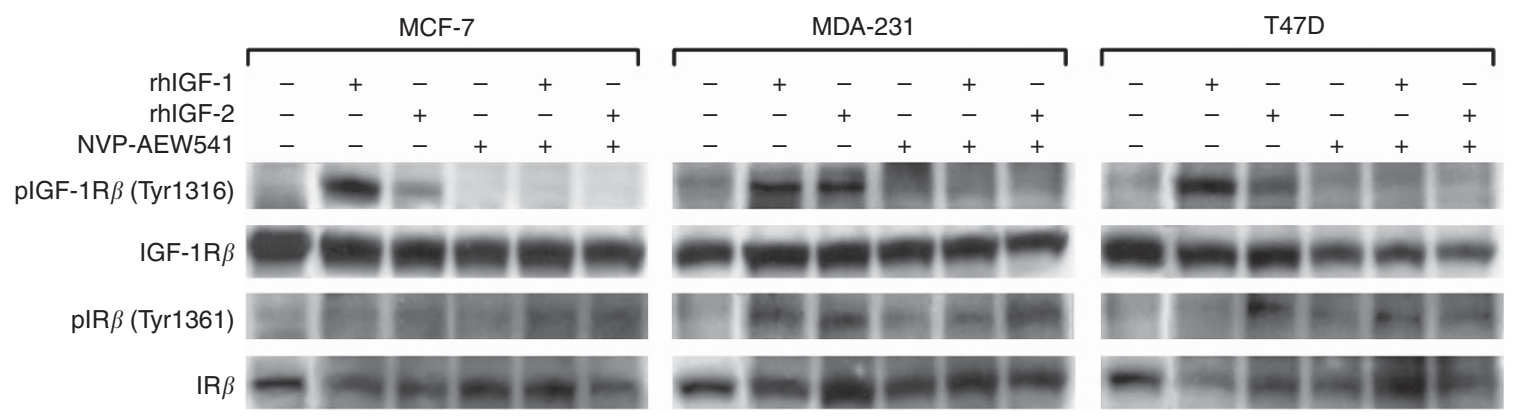

Figure 2. Effect of NVP-AEW541 ( $1 \mu \mathrm{M}$ for $24 \mathrm{~h}$ ) on total and phosphorylated IGF-1R and IR levels in MCF7, MDA-231 and T47D cells, in the absence of stimulation or after exposure to rhIGF-1 or rhIGF-2 (50 $\mathrm{n} \mathrm{ml}^{-1}$ in serum-free medium for 15 min: cells were grown in serum-free medium for the last $6 \mathrm{~h}$ of treatment before exposure to IGFs). Actin levels were simultaneously assessed as an internal loading control. 
A
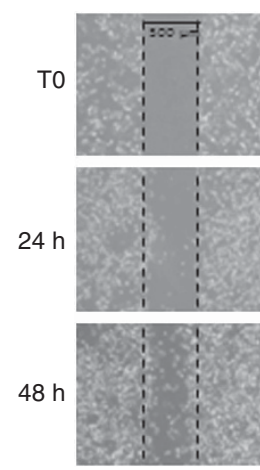

B
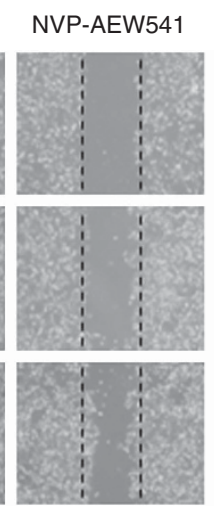

+ rhIGF-1
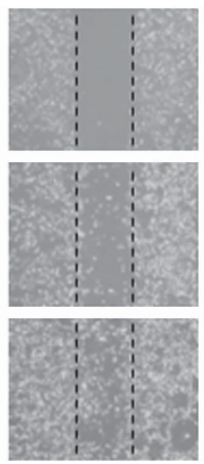

+ rhIGF-1 + NVP-AEW541
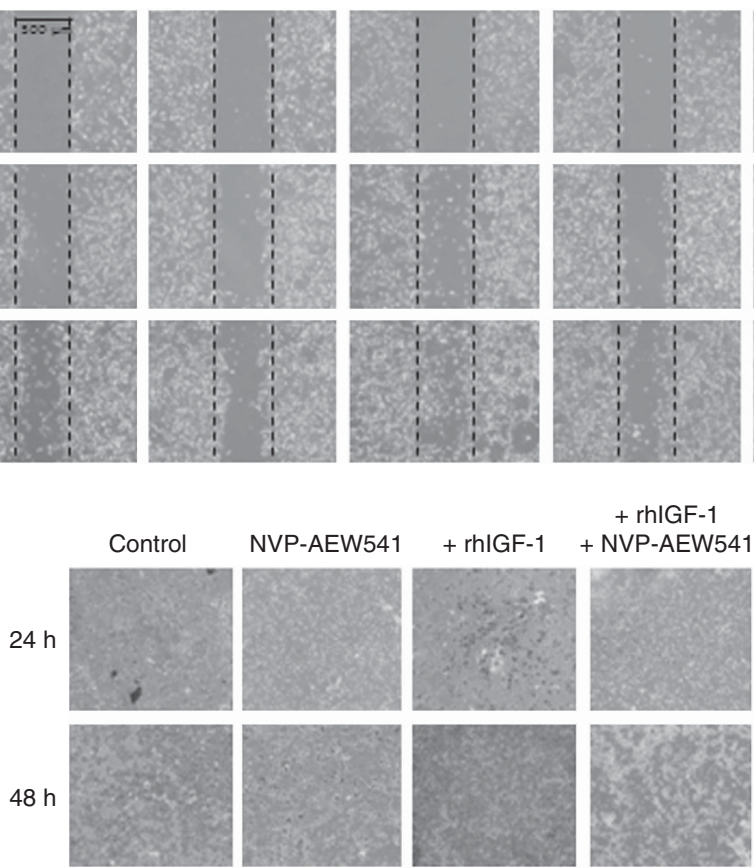

+ rhIGF-1 + NVP-AEW541
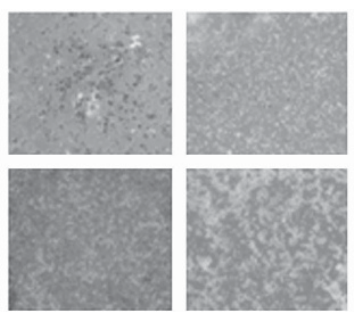

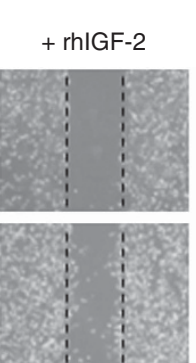

+ rhIGF-2 + NVP-AEW541
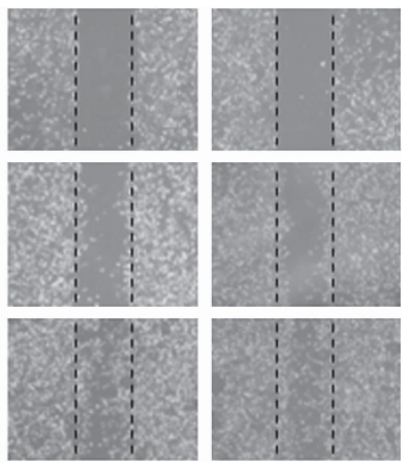

+ rhIGF-2 + NVP-AEW541
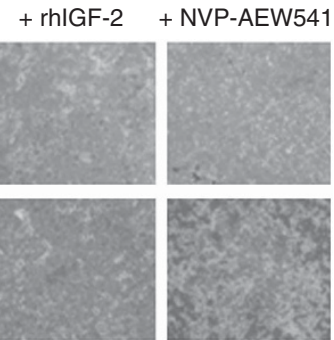

Figure 3. Effect of NVP-AEW541 (1 $\mu \mathrm{m})$ on the IGF-induced migration of MDA-231 cells, as assessed by the scratch wound-healing and Boyden chamber assays. (A) Cells were grown in specific supports for $24 \mathrm{~h}$, then the inserts were removed and the medium was replaced with serum-free medium containing NVP-AEW541 with or without rhIGF-1 or rhIGF2 $\left(50 \mathrm{ng} \mathrm{ml}^{-1}\right)$. Pictures were taken at the time of insert removal (T0) and at regular time intervals, up to $48 \mathrm{~h}$. (B) Cells, previously starved for $24 \mathrm{~h}$, were added on top of pre-incubated Boyden chambers and allowed to grow and migrate (in the presence of NVP-AEW541 with or without $50 \mathrm{ng} \mathrm{ml}^{-1}$ of rhIGF-1 or rhlGF-2) for 24 or $48 \mathrm{~h}$ : the cells able to migrate in the chamber were stained and photographed, and differences between the treatments were assessed by visual inspection.

HIF-1 inhibition decreases IGF-2-dependent signalling and enhances the effects of NVP-AEW541 on cell migration. Given the ability of hypoxia to undermine the ability of selective IGF-1R inhibition to impair cell migration through HIF-1-dependent IGF-2 release, we finally evaluated the efficacy of the combination of NVP-AEW541 with HIF-1 inhibition in MDA-231 cells.

To downregulate HIF-1 activity, we used topotecan, a DNA topoisomerase I poison that has been reported to inhibit the translation of HIF- $1 \alpha$ mRNA (Onnis et al, 2009). The results of western blotting analysis confirm the effectiveness of topotecan as a HIF- $1 \alpha$ inhibitor: as expected, and as shown in Figures $5 \mathrm{~A}$ and $6 \mathrm{~A}, \mathrm{HIF}-1 \alpha$ protein levels were consistently decreased following 24 -h exposure to $250 \mathrm{~nm}$ of topotecan under hypoxia; this topotecan concentration was chosen based on a preliminary cytotoxicity assay, indicating that $<10 \%$ of the cells were killed by the drug under our experimental conditions. (Supplementary Figure S9, panel a). As a further corroboration of topotecaninduced HIF-1 inhibition, real time-PCR analysis showed that the mRNA levels of three HIF-1-regulated genes, namely, IGF-2, $V E G F A$ and $P D K-1$, were restored to almost normoxic levels (Supplementary Figure S9, panel b) following topotecan treatment. It should be emphasised that, while topotecan alone caused a decrease in phosphorylation of both receptors, the topotecan/NVP-AEW541 combination achieved near-complete abrogation (Figure 6A; densitometric analyses are reported in Supplementary Figure S10). Accordingly, topotecan, both as a single agent and in combination with NVP-AEW541, was able to curtail the migration of MDA-231 cells in both assays (Figure 6B and $\mathrm{C}$ ). Topotecan and the topotecan/NVP-AEW541 combination were observed to exert similar effects on hypoxia-induced migration of MDA-468 cells (Supplementary Figure S11), suggesting that our findings might be extended to other TNBC models.

\section{DISCUSSION}

The IGF axis and, more specifically, IGF-1R have long been considered as attractive targets for the development of novel anticancer therapies. Besides playing a crucial role in regulating cell growth, proliferation and differentiation, IGF-1R and its downstream signalling pathways have been implicated in the development of resistance to endocrine and targeted therapies (Gao et al, 2012; Karamouzis and Papavassiliou, 2012). In addition, a role in tumour cell migration and metastatic potential and a direct involvement in the metastatic cascade in breast have recently been proposed (Zhu et al, 2011). Interestingly, IGF-dependent enhanced cellular proliferation has been documented in TNBCs, which can provide new targets for the so far limited therapeutic options of this sub-type that lacks expression of other druggable targets, such as ER or HER-2 (Davison et al, 2011).

In the present study, we have focused on the role of the IGF/ insulin axis on cell migration and on the possibility to suppress this phenotype using NVP-AEW541, a selective IGF-1R inhibitor (García-Echeverría et al, 2004), in MDA-231 cells, a human breast cancer cell line derived from a mTNBC. Two other cell lines, ERpositive MCF-7 and T47D, were also examined, but although both were shown to express IGF-1R and IR, neither displayed a migratory response to exogenous rhIGF-1 or rhIGF-2. Our results indicate that, while NVP-AEW541 effectively inhibits IGF-1R phosphorylation in response to both exogenous IGFs, only rhIGF1 -induced migration is significantly reduced by this compound, whereas the effect of rhIGF-2 on cell migration is remarkably resistant to this approach. Several different drug candidates targeting IGF-1R, including both anti-receptor antibodies and small-molecule receptor kinase inhibitors, have shown activity in model systems, and the former (e.g., figitumumab, ganitumumab, 


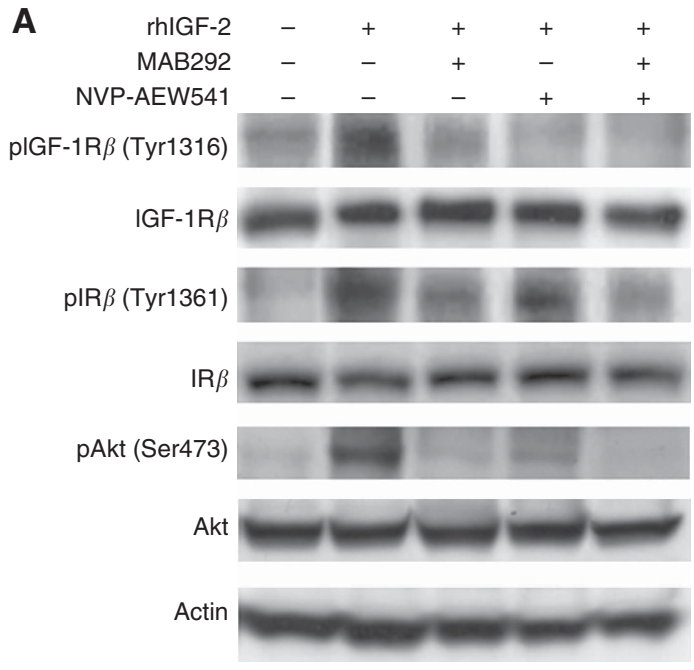

B
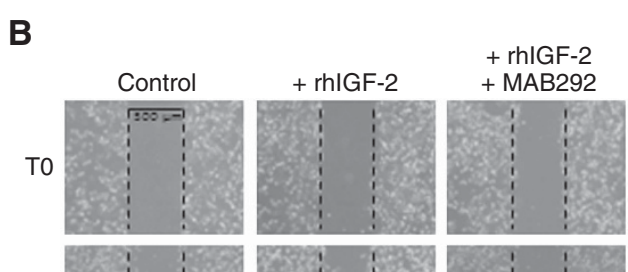

+ rhIGF-2
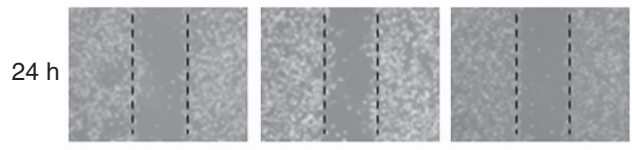

政W 54
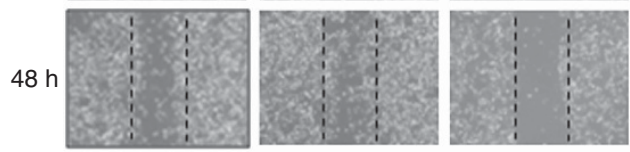

+ MAB292

C

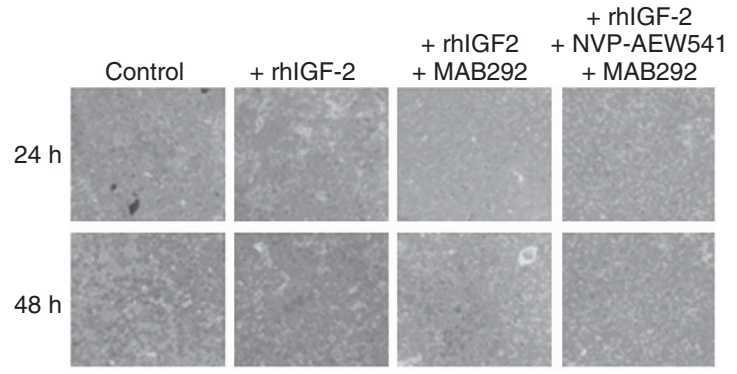

Figure 4. Inhibition of IGF-2 signalling by the specific monoclonal antibody MAB292 enhances the anti-migratory effect of NVP-

AEW541. (A) Comparative effects of MAB292 $\left(5 \mu \mathrm{g} \mathrm{ml}^{-1}\right.$ for $\left.24 \mathrm{~h}\right)$ and NVP-AEW541 ( $1 \mu \mathrm{m}$ for $24 \mathrm{~h}$ ) on IGF-1R, IR and Akt phosphorylation in rhIGF-2-stimulated MDA-231 cells $\left(50 \mathrm{ng} \mathrm{ml}^{-1}\right.$ in serum-free medium for $15 \mathrm{~min}$, following $6 \mathrm{~h}$ of starvation). Actin levels were simultaneously assessed as an internal control. (B and C) Comparative effects of MAB292 (5 $\left.\mathrm{g} \mathrm{ml}^{-1}\right)$ and NVP-AEW541 (1 $\left.\mu \mathrm{M}\right)$ on rhIGF-2induced migration of MDA-231 cells as assessed by the scratch woundhealing (B) and Boyden chamber (C) assays. The experiments were performed according to the same protocol as in Figure $3 \mathrm{~A}$ and $\mathrm{B}$.

cixutumumab) have been the subject of intense clinical research. Although some Phase II studies of IGF-1R-specific antibodies showed activity with little toxicity, and there were reports of major responses, Phase III trial reports have documented a lack of efficacy, together with significant metabolic toxicity (chiefly hyperglycaemia; Pollak, 2008a; Gao et al, 2012; Karamouzis and Papavassiliou, 2012). Although systemic compensatory feedback mechanisms may account, at least in part, for the observed failure of agents specifically targeting IGF-1R in the clinical settings, our data indicate that compensatory mechanisms can also operate at the cellular level, significantly hindering the anti-migratory effect of NVP-AEW541 in our experimental setup. Our working hypothesis was that interaction of IGFs, especially IGF-2, with IR (which is not targeted by NVP-AEW541) might explain the failure of this compound to suppress breast cancer cell migration. MDA231 cells do, in fact, express both IR-A and IR-B, and western blotting analysis indicates that NVP-AEW541 does not affect IGF2 -induced IR phosphorylation, nor is it able to completely abolish downstream signalling, as shown by residual activation of Akt. Rather more surprisingly, we also observed some degree of IR activation in cells stimulated with IGF-1, which was not prevented by NVP-AEW541 exposure; however, this finding could be due to the use of supraphysiological ligand concentrations, which might engage a significant fraction of IRs in spite of low affinity, while IGF-2 remains the most likely IR activator under physiological conditions. Alternatively, supraphysiological IGF-1 concentrations might cause indirect IR activation by increasing intracellular HIF$1 \alpha$ levels, as observed in experimental models derived from different tumour types (Stoeltzing et al, 2003; Thomas and Kim, 2009; Gariboldi et al, 2010), and this in turn would cause the release of endogenous IGF-2. Dual IGF-1R/IR inhibition could prevail over this signal redundancy, but the role played by IR in cellular metabolism raises serious concerns regarding the safety of this approach in the clinic. The existence of two IR variants, the former of which (A) is preferentially expressed in tumours, whereas variant $\mathrm{B}$ is thought to have the major role in glucose metabolism, provides a window of opportunity for intervention specifically directed at tumours (Buck et al, 2010); however, the fact that the two variants only differ in the presence/absence of exon 11 (coding for a fragment of 12 aa in the C-terminal portion of the $\alpha$ subunit of the receptor) makes the design of variantselective inhibitors and antibodies particularly challenging.

In order to simultaneously inhibit activation of both IGF-1R and IR by IGF-2, a different and probably more feasible strategy would be to block IGF-2 itself. IGF-2 has been reported to selectively activate IR-A in several tumour tissues, whereas its binding affinity for IR-B is negligible (Frasca et al, 1999; Pollak, 2012). Thus, sequestering IGF- 2 would abrogate signalling through both IGF-1R and IR-A, as well as through their hybrid isoforms, all of which are frequently overexpressed in cancer cells, while sparing insulin-dependent signalling through IR-B, thereby reducing the risk of metabolic side-effects. MEDI-573 is the only antibody currently in clinical testing that exerts its effects by neutralising not only IGF-2 but also IGF-1, thus inhibiting IGF signalling through IGF-1R, IR and their hybrid receptors. The ongoing Phase I clinical trial has shown promising results and strongly suggests that MEDI573 might achieve this result without inducing hyperglycaemia (Gao et al, 2011). If confirmed, this observation would be consistent with better expectations of ligand- vs receptor-based targeting of the IGF axis. Our data obtained with a neutralising antibody specific for IGF-2 (MAB292) seem to support the involvement of IGF-2-induced IGF-1R/IR activation in the poor anti-migratory effect of NVP-AEW541. In MDA-231 cells exposed to IGF-2, MAB292 was able to prevent phosphorylation of both receptors; more significantly, stimulation of cell migration by rhIGF- 2 was dramatically reduced by MAB292 and completely abolished when the antibody was combined with NVPAEW541.

IGF-2 neutralisation could be a particularly winning strategy, especially considering the fact that, at low oxygen levels that are frequently encountered within the tumour mass $\left(\mathrm{pO}_{2}<2.5 \%\right)$, HIF- $1 \alpha$ stabilisation and subsequent dimerisation with HIF-1 $\beta$ leads to increased IGF-2 levels, as reported in the literature (Feldser et al, 1999; Semenza, 2012) and as observed both at the transcript and protein levels in the present experiments on MDA-231 cells. 


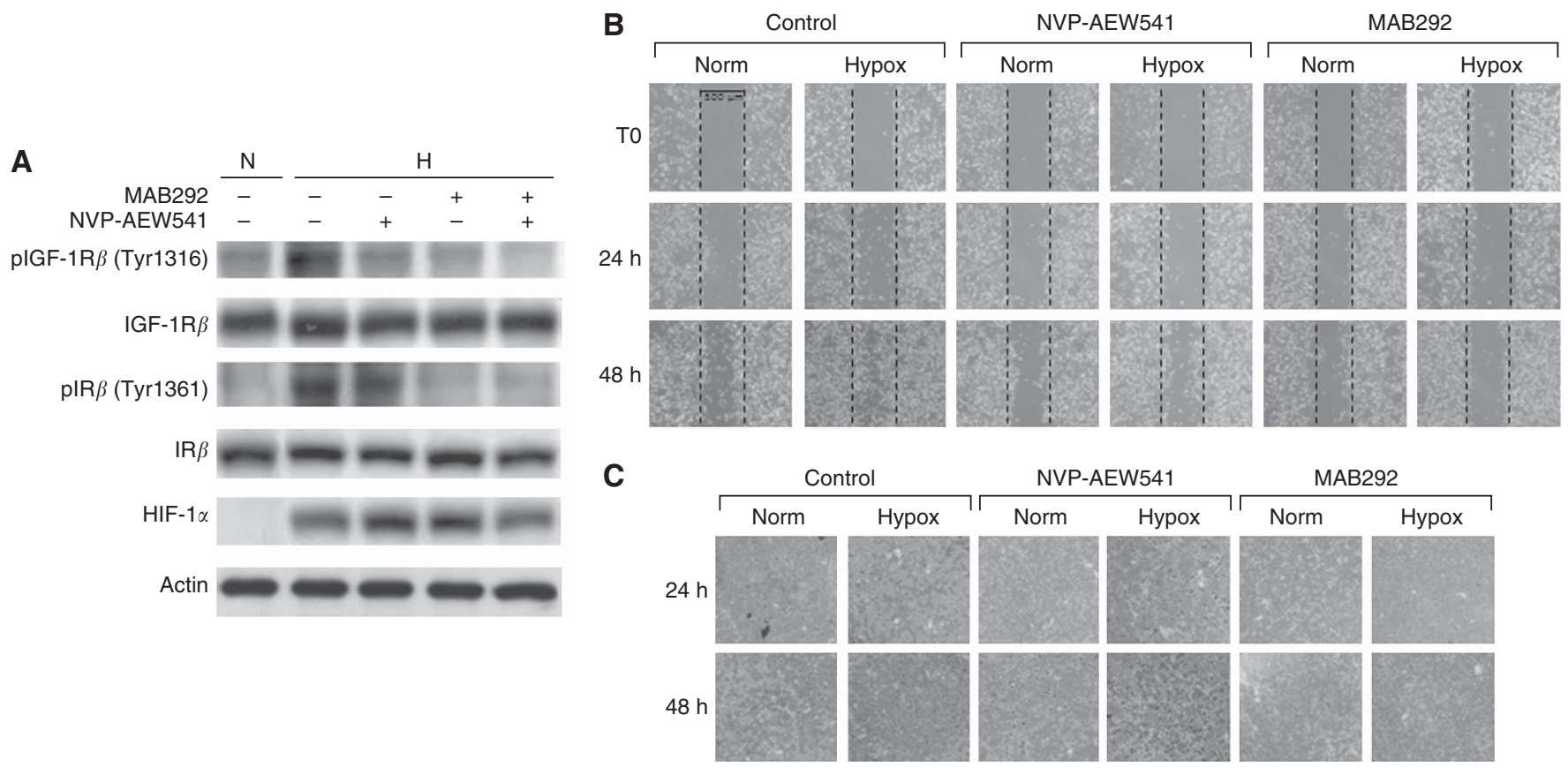

Figure 5. HIF-1 $\alpha$ stabilisation decreases the anti-migratory effect of NVP-AEW541. (A) Effect of HIF-1 $\alpha$ stabilisation under hypoxic conditions ( $\mathrm{pO}{ }_{2}$ $1 \%)$ on total and phosphorylated IGF-1R and IR levels in MDA-231 cells, and effect of MAB292 (5 $\mu \mathrm{g} \mathrm{ml}^{-1}$ for $\left.24 \mathrm{~h}\right)$ and NVP-AEW541 (1 $\mu \mathrm{M}$ for $24 \mathrm{~h}$ ) on IGF-R1 and IR phosphorylation. Cells were grown for $24 \mathrm{~h}$ under normoxic conditions $\left(\mathrm{pO}_{2} 21 \%\right)$ and subsequently incubated under hypoxia for $24 \mathrm{~h}$, with or without MAB292 or NVP-AEW541. Normoxic controls were also included for comparison; actin levels were simultaneously assessed as an internal loading control. (B and C) Comparative effects of MAB292 (5 $\left.\mathrm{g} \mathrm{ml}^{-1}\right)$ and NVP-AEW541 (1 $\left.\mu \mathrm{M}\right)$ in inhibiting the migration of unstimulated MDA231 cells, as assessed by the scratch wound-healing (B) and Boyden chamber (C) assays. Hypoxia was started at T0 following 24-h incubation under normoxic conditions; the experiments were performed according to the same protocol as in Figure 3A and B.

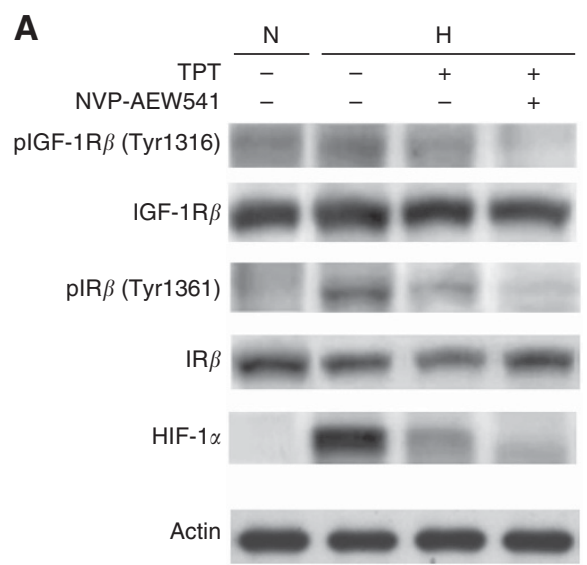

B

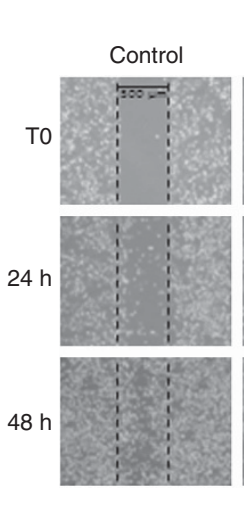

C

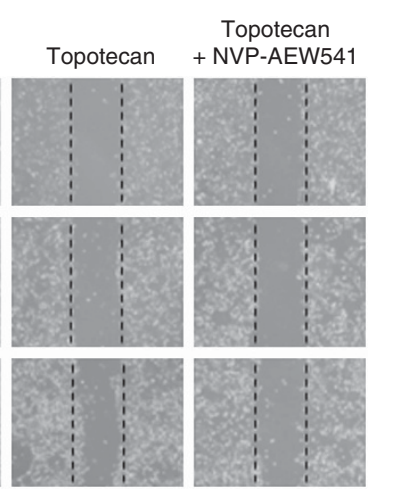

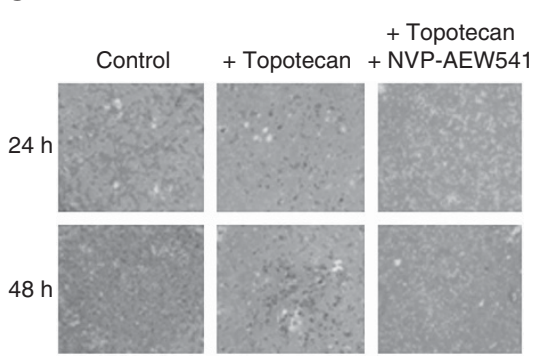

Figure 6. HIF-1 inhibition decreases IGF-2-dependent signalling and enhances the anti-migratory effect of NVP-AEW541. (A) Effect of topotecan (TPT; $250 \mathrm{~nm}$ for $24 \mathrm{~h}$ ) on HIF-1 $\alpha$ levels and on total and phosphorylated IGF-1R and IR in MDA-231 cells under hypoxic conditions ( $\mathrm{pO} 2 \mathrm{2} \%$ ), alone or in combination with NVP-AEW541 ( $1 \mu \mathrm{M}$ for $24 \mathrm{~h}$ ), as assessed by western blotting. Cells were grown for $24 \mathrm{~h}$ under normoxic conditions $\left(\mathrm{pO}_{2} 21 \%\right)$ and subsequently incubated under hypoxia for $24 \mathrm{~h}$, with or without TPT \pm NVP-AEW541. Normoxic controls were also included for comparison; actin levels were simultaneously assessed as an internal loading control. (B and C) Effect of TPT (250 nM), alone or in combination with NVP-AEW541 $(1 \mu \mathrm{M})$, on the migration of unstimulated MDA-231 cells, as assessed by the scratch wound-healing (B) and Boyden chamber (C) assays. Hypoxia was started at T0, following 24-h incubation under normoxic conditions; the experiments were performed according to the same protocol as in Figure $3 \mathrm{~A}$ and $\mathrm{B}$.

This HIF-1-dependent increase in IGF-2 release possibly explains why NVP-AEW541 fails to block MDA-231 cell migration, similarly to what has been observed in the presence of exogenous IGF-2 (although obviously to a lesser extent, considering the lower concentration of IGF-2 released under hypoxia as compared with the one administered exogenously). In support of the key role played by IGF-2 in obstructing the anti-migratory effect of NVPAEW541 following HIF- $1 \alpha$ stabilisation, addition of MAB292 is as effective in inhibiting the migration of hypoxic MDA-231 cells as it is following exposure to exogenous rhIGF-2.

Based on this observation, we suggest that a novel strategy to overcome IR stimulation by IGF-2 following IGF-1R inhibition may be simultaneous HIF- $1 \alpha$ inhibition. The discovery of HIF-1 (and later HIF-2) and its role in cancer cell proliferation and survival in conditions of reduced oxygenation $\left(\mathrm{pO}_{2}<2.5 \%\right)$ has led to a great interest in the development of HIF- $1 \alpha$ and HIF- $2 \alpha$ 
inhibitors. At present, the major problem in targeting HIFs is the lack of specific inhibitors: many chemotherapeutic drugs, in fact, have a complete or partial inhibitory activity on HIF- $1 \alpha$, but this is often accompanied by a number of off-target effects. Specific inhibitors of HIF- $1 \alpha$ and/or HIF- $2 \alpha$ are currently under validation (Onnis et al, 2009; Xia et al, 2012).

In our study, HIF- $1 \alpha$ inhibition was achieved using topotecan, a topoisomerase I poison with major clinical applications in the management of ovarian and small cell lung cancer. Topotecan inhibits HIF- $1 \alpha$ translation through a topoisomerase I-dependent but DNA damage-independent mechanism, suggesting that HIF- $1 \alpha$ inhibition could be mechanistically distinct from cytotoxicity (Onnis et al, 2009). In our experiments, topotecan (used at subtoxic concentrations, so that HIF- $1 \alpha$ inhibition could predominate over the cytotoxic effect of the drug) was confirmed to decrease HIF- $1 \alpha$ protein levels, as well as the expression of different HIF-1 target genes (IGF2, VEGFA and PDK1). As a result of the decrease in IGF-2 release, phosphorylation of both IR and IGF-1R was reduced by topotecan treatment and nearly abrogated by concurrent treatment with topotecan and NVP-AEW541; importantly, the effect of NVP-AEW541 on migration of MDA-231 cells under hypoxia was restored to normoxic levels (or lower). Overall, we can conclude that combined inhibition of IGF-1R and HIF-1 appears to result in a synergistic effect on the migration of triple-negative MDA-231 breast cancer cells, and similar results obtained in MDA-468 cells, also triple-negative, raise the hope that our findings may be extended to this hard-to-treat breast cancer subtype. Thus, inhibiting the IGF and HIF systems during the early stages of breast cancer might provide a promising therapeutic option, most notably for TNBC, by preventing progression to MBC, besides reducing the contribution of these systems to the development of resistance against both cytotoxic and targeted agents.

\section{REFERENCES}

Alvarez RH, Valero V, Hortobagyi GN (2010) Emerging targeted therapies for breast cancer. J Clin Oncol 28(20): 3366-3379.

Belfiore A (2007) The role of insulin receptor isoforms and hybrid insulin/ IGF-I receptors in human cancer. Curr Pharm Des 13(7): 671-686.

Beslija S, Bonneterre J, Burstein HJ, Cocquyt V, Gnant M, Heinemann V, Jassem J, Köstler WJ, Krainer M, Menard S, Petit T, Petruzelka L, Possinger K, Schmid P, Stadtmauer E, Stockler M, Van Belle S, Vogel C, Wilcken N, Wiltschke C, Zielinski CC, Zwierzina H. (CECOG) CECOG (2009) Third consensus on medical treatment of metastatic breast cancer. Ann Oncol 20(11): 1771-1785.

Buck E, Gokhale PC, Koujak S, Brown E, Eyzaguirre A, Tao N, Rosenfeld-Franklin M, Lerner L, Chiu MI, Wild R, Epstein D, Pachter JA Miglarese MR (2010) Compensatory insulin receptor (IR) activation on inhibition of insulin-like growth factor-1 receptor (IGF-1 R): rationale for cotargeting IGF-1R and IR in cancer. Mol Cancer Ther 9(10): 2652-2664.

Davison Z, de Blacquière GE, Westley BR, May FE (2011) Insulin-like growth factor-dependent proliferation and survival of triple-negative breast cancer cells: implications for therapy. Neoplasia 13(6): 504-515.

Feldser D, Agani F, Iyer N, Pak B, Ferreira G, Semenza G (1999) Reciprocal positive regulation of hypoxia-inducible factor 1alpha and insulin-like growth factor 2. Cancer Res 59(16): 3915-3918.

Frasca F, Pandini G, Scalia P, Sciacca L, Mineo R, Costantino A, Goldfine ID, Belfiore A, Vigneri R (1999) Insulin receptor isoform A, a newly recognized, high-affinity insulin-like growth factor II receptor in fetal and cancer cells. Mol Cell Biol 19(5): 3278-3288.

Gao J, Chang YS, Jallal B, Viner J (2012) Targeting the insulin-like growth factor axis for the development of novel therapeutics in oncology. Cancer Res 72(1): 3-12

Gao J, Chesebrough JW, Cartlidge SA, Ricketts SA, Incognito L, Veldman-Jones M, Blakey DC, Tabrizi M, Jallal B, Trail PA, Coats S, Bosslet K, Chang YS (2011) Dual IGF-I/II-neutralizing antibody MEDI-573 potently inhibits IGF signaling and tumor growth. Cancer Res 71(3): 1029-1040.

García-Echeverría C, Pearson M, Marti A, Meyer T, Mestan J, Zimmermann J, Gao J, Brueggen J, Capraro H, Cozens R, Evans D, Fabbro D, Furet P,
Porta D, Liebetanz J, Martiny-Baron G, Ruetz S, Hofmann F (2004) In vivo antitumor activity of NVP-AEW541-A novel, potent, and selective inhibitor of the IGF-IR kinase. Cancer Cell 5(3): 231-239.

Gariboldi M, Ravizza R, Monti E (2010) The IGFR1 inhibitor NVP-AEW541 disrupts a pro-survival and pro-angiogenic IGF-STAT3-HIF1 pathway in human glioblastoma cells. Biochem Pharmacol 80(4): 455-462.

Guarneri V, Conte P (2009) Metastatic breast cancer: therapeutic options according to molecular subtypes and prior adjuvant therapy. Oncologist 14(7): 645-656.

Hankinson SE, Willett WC, Colditz GA, Hunter DJ, Michaud DS, Deroo B, Rosner B, Speizer FE, Pollak M (1998) Circulating concentrations of insulin-like growth factor-I and risk of breast cancer. Lancet 351(9113): 1393-1396.

Helle SI (2004) The insulin-like growth factor system in advanced breast cancer. Best Pract Res Clin Endocrinol Metab 18(1): 67-79.

Karamouzis MV, Papavassiliou AG (2012) Targeting insulin-like growth factor in breast cancer therapeutics. Crit Rev Oncol Hematol 84(1): 8-17.

Liedtke C, Kiesel L (2011) Current issues of targeted therapy in metastatic triple-negative breast cancer. Breast Care (Basel) 6(3): 234-239.

Martin JL, Baxter RC (2011) Signalling pathways of insulin-like growth factors (IGFs) and IGF binding protein-3. Growth Factors 29(6): 235-244.

McCann AH, Miller N, O’Meara A, Pedersen I, Keogh K, Gorey T, Dervan PA (1996) Biallelic expression of the IGF2 gene in human breast disease. Hum Mol Genet 5(8): 1123-1127.

Onnis B, Rapisarda A, Melillo G (2009) Development of HIF-1 inhibitors for cancer therapy. J Cell Mol Med 13(9A): 2780-2786.

Pandini G, Vigneri R, Costantino A, Frasca F, Ippolito A, Fujita-Yamaguchi Y, Siddle K, Goldfine ID, Belfiore A (1999) Insulin and insulin-like growth factor-I (IGF-I) receptor overexpression in breast cancers leads to insulin/ IGF-I hybrid receptor overexpression: evidence for a second mechanism of IGF-I signaling. Clin Cancer Res 5(7): 1935-1944.

Pollak M (2008a) Insulin and insulin-like growth factor signalling in neoplasia. Nat Rev Cancer 8(12): 915-928.

Pollak M (2008b) Insulin and insulin-like growth factor signalling in neoplasia. Nat Rev Cancer 8(12): 915-928.

Pollak M (2012) The insulin and insulin-like growth factor receptor family in neoplasia: an update. Nat Rev Cancer 12(3): 159-169.

Reis-Filho J, Tutt A (2008) Triple negative tumours: a critical review. Histopathology 52(1): 108-118.

Richardson AE, Hamilton N, Davis W, Brito C, De León D (2011) Insulin-like growth factor-2 (IGF-2) activates estrogen receptor- $\alpha$ and $-\beta$ via the IGF-1 and the insulin receptors in breast cancer cells. Growth Factors 29(2-3): 82-93.

Sachdev D, Yee D (2001) The IGF system and breast cancer. Endocr Relat Cancer 8(3): 197-209.

Sachdev D, Yee D (2007) Disrupting insulin-like growth factor signaling as a potential cancer therapy. Mol Cancer Ther 6(1): 1-12.

Sachdev D, Zhang X, Matise I, Gaillard-Kelly M, Yee D (2010) The type I insulin-like growth factor receptor regulates cancer metastasis independently of primary tumor growth by promoting invasion and survival. Oncogene 29(2): 251-262.

Saldana SM, Lee HH, Lowery FJ, Khotskaya YB, Xia W, Zhang C, Chang SS, Chou CK, Steeg PS, Yu D, Hung MC (2013) Inhibition of type I insulinlike growth factor receptor signaling attenuates the development of breast cancer brain metastasis. PLoS One 8(9): e73406.

Semenza GL (2012) Hypoxia-inducible factors: mediators of cancer progression and targets for cancer therapy. Trends Pharmacol Sci 33(4): 207-214.

Stoeltzing O, Liu W, Reinmuth N, Fan F, Parikh A, Bucana C, Evans D, Semenza G, Ellis L (2003) Regulation of hypoxia-inducible factor-1alpha, vascular endothelial growth factor, and angiogenesis by an insulin-like growth factor-I receptor autocrine loop in human pancreatic cancer. Am J Pathol 163(3): 1001-1011.

Sánchez-Muñoz A, Pérez-Ruiz E, Jiménez B, Ribelles N, Márquez A, García-Ríos I, Alba Conejo E (2009) Targeted therapy of metastatic breast cancer. Clin Transl Oncol 11(10): 643-650.

Thomas R, Kim M (2009) A HIF-1alpha-dependent autocrine feedback loop promotes survival of serum-deprived prostate cancer cells. Prostate 69(3): 263-275.

Vaupel P, Schlenger K, Knoop C, Höckel M (1991) Oxygenation of human tumors: evaluation of tissue oxygen distribution in breast cancers 
by computerized $\mathrm{O} 2$ tension measurements. Cancer Res 51(12): 3316-3322.

Vleugel MM, Greijer AE, Shvarts A, van der Groep P, van Berkel M, Aarbodem Y, van Tinteren H, Harris AL, van Diest PJ, van der Wall E (2005) Differential prognostic impact of hypoxia induced and diffuse HIF1alpha expression in invasive breast cancer. J Clin Pathol 58(2): 172-177.

Xia Y, Choi HK, Lee K (2012) Recent advances in hypoxia-inducible factor (HIF)-1 inhibitors. Eur J Med Chem 49: 24-40.

Zhang H, Fagan DH, Zeng X, Freeman KT, Sachdev D, Yee D (2010) Inhibition of cancer cell proliferation and metastasis by insulin receptor downregulation. Oncogene 29(17): 2517-2527.

Zhang H, Pelzer AM, Kiang DT, Yee D (2007) Down-regulation of type I insulin-like growth factor receptor increases sensitivity of breast cancer cells to insulin. Cancer Res 67(1): 391-397.
Zhang Y, Moerkens M, Ramaiahgari S, de Bont H, Price L, Meerman J, van de Water B (2011) Elevated insulin-like growth factor 1 receptor signaling induces antiestrogen resistance through the MAPK/ERK and PI3K/Akt signaling routes. Breast Cancer Res 13(3): R52.

Zhu C, Qi X, Chen Y, Sun B, Dai Y, Gu Y (2011) PI3K/Akt and MAPK/ERK1/ 2 signaling pathways are involved in IGF-1-induced VEGF-C upregulation in breast cancer. J Cancer Res Clin Oncol 137(11): 1587-1594.

This work is published under the standard license to publish agreement. After 12 months the work will become freely available and the license terms will switch to a Creative Commons AttributionNonCommercial-Share Alike 3.0 Unported License.

Supplementary Information accompanies this paper on British Journal of Cancer website (http://www.nature.com/bjc) 